\title{
Prefrontal Cortex Lesions Disrupt the Contextual Control of Response Conflict
}

\author{
Josephine E. Haddon and Simon Killcross \\ School of Psychology, Cardiff University, Cardiff CF10 3AT, United Kingdom
}

The prefrontal cortex has been implicated in multiple forms of goal-directed behavior. Rats with pretraining lesions to the prefrontal cortex (PFC) or specific lesions to the anterior cingulate cortex (ACC) were trained and tested on a novel behavioral procedure measuring aspects of cue and response competition typical of tests of prefrontal function in humans. Rats were trained on two biconditional discrimination tasks, one auditory and one visual, in two discriminably different contexts. At test, they received presentations of audiovisual compounds of these training stimuli in both contexts, in extinction. These compounds were formed in such way that the individual elements had dictated either the same (congruent trials) or different (incongruent trials) responses during training. Sham-operated rats used the contextual cues to disambiguate the conflicting response information provided by incongruent stimulus compounds. ACC lesions impaired the contextual control of instrumental responding during incongruent cues during only the initial period of cue presentation, whereas larger PFC lesions abolished incongruent cue performance completely. Neither biconditional discrimination acquisition, nor test performance during congruent stimulus compounds, were affected by the lesions. These findings are consistent with human and nonhuman primate studies, indicating a role for the PFC in the processes by which cues come to control behavior in the face of conflicting information and the ACC specifically in processes such as detection of response conflict. This procedure provides a good foundation for an improved understanding of the disruption to goal-directed behavior seen with frontal dysfunction in a number of neuropsychological disorders including schizophrenia.

Key words: Stroop; conflict; prefrontal; prelimbic; goal directed; context

\section{Introduction}

Cohen and colleagues (Cohen et al., 1990; Cohen and ServenSchreiber, 1992; Miller and Cohen, 2001) proposed a model of goal-directed behavior in which task-specific information is used for the resolution of cue and response conflict. This model proposes that a particular task (or goal) choice is specified by the pattern of activation over a set of instructional inputs, termed "contextual" units. Activation of these task-appropriate units modulates the activity of competing stimulus-response pathways, favoring the pathway relevant to the current task over alternative pathways.

This cognitive control is thought to be vital in situations when there is conflict between responses (response competition), when a goal or task instruction has to be maintained over time (working memory), or where one has to switch between a number of possible alternatives (Cohen et al., 1998; Miller and Cohen, 2001). Response competition may occur in situations in which there are ambiguous stimuli, in which multiple responses are possible, or when a dominant stimulus-response pathway needs

Received Aug. 3, 2005; revised Jan. 26, 2006; accepted Jan. 26, 2006

This work was supported by an Independent Investigator award (Southwest Florida named award) from the National Alliance for Research into Schizophrenia and Depression (S.K.) and by a Biotechnology and Biological Sciences Research Council Co-operative Award in Science and Engineering in conjunction with Merck, Sharp, and Dohme (J.E.H.).

Correspondence should be addressed to Simon Killcross, School of Psychology, Cardiff University, Tower Building, Park Place, Cardiff CF10 3AT, UK. E-mail: KillcrossAS@cardiff.ac.uk.

DOI:10.1523/JNEUROSCI.3243-05.2006

Copyright $\odot 2006$ Society for Neuroscience $\quad$ 0270-6474/06/262933-08\$15.00/0 to be suppressed. For example, contextual control is thought to be essential for the performance of a number of cognitive paradigms such as the continuous performance task (Rosvold et al., 1956), task switching (Wylie and Allport, 2000), and the Stroop task (Stroop, 1935; MacLeod, 1991).

The prefrontal cortex (PFC) is likely to be responsible for guiding behavior in such situations of cue and response competition (Miller and Cohen, 2001). Furthermore, some researchers have suggested a more specific role for the anterior cingulate cortex (ACC) in the detection of response conflict (Carter et al., 1998, 2000; Botvinick et al., 1999). This conflict detection signal is thought to be important in directing trial-by-trial adjustments in the amount of control provided by the prefrontal cortex (or by contextual control units in the network model described above) (Miller and Cohen, 2001).

Recently, we presented a task designed to mimic some aspects of cue and response competition seen in tasks examining cognitive control (J. E. Haddon, D. N. George, and S. Killcross, unpublished observations). In this task, rats were trained on two instrumental biconditional discrimination tasks, one auditory and one visual, in two different contexts. At test, audiovisual compounds of the training stimuli were presented. These compounds comprised stimulus elements that either dictated the same instrumental response during training or different lever press responses during training (termed congruent and incongruent compounds, respectively). We showed that responding to incongruent compounds was controlled by the contextual cues present 
during the test session, with rats biasing their responses according to the stimulus element previously trained in the test context. Thus, the context came to disambiguate the conflicting information provided by incongruent stimulus compounds.

If the cognitive control of responding by contextual cues observed with this paradigm parallels the way in which task instructions disambiguate conflicting response information in cognitive control tasks, then one might expect that this higher-order, rulebased process may be dependent on the integrity of the prefrontal cortex. In contrast, if the contextual control of incongruent compounds is more akin to the contextual modulation of simple associations (i.e., context specific latent inhibition), then it is unlikely that prefrontal damage will impact on performance of this task. We examined the effect of lesions of the prefrontal cortex and to the anterior cingulate cortex on this contextual control of conditional instrumental responding.

\section{Materials and Methods}

\section{Experiment 1a: contextual control of biconditional task performance}

Subjects

Thirty-six naive, adult, male hooded Lister rats (Harlan Olac, Bicester, UK) served in this experiment. Twelve of the rats received pretraining excitotoxic lesions of the PFC, 12 received pretraining excitotoxic lesions of the ACC, and the remaining 12 served as sham-operated controls. After surgery, the rats were maintained at $85 \%$ of their age-matched ad libitum weights (range, 300-350 g) and had ad libitum access to water. The colony room housing the rats operated on a $12 \mathrm{~h}$ light/dark cycle (lights on at 8:00 A.M.) and was maintained at a temperature of $21 \pm 1^{\circ} \mathrm{C}$ and humidity of $55 \pm 5 \%$. The rats were housed in pairs.

\section{Surgery}

Rats were anesthetized with isoflurane, their heads shaved, and placed in a stereotaxic frame (Kopf Instruments, Tujunga, CA). An incision was made in the scalp, and then a skull flap overlying the prefrontal cortex was drilled out. For lesions within the prefrontal cortex, injections of ibotenic acid ( $63 \mathrm{~mm}$; Sigma, Poole, UK) were made with a $2 \mu$ l Hamilton (Reno, NV) syringe mounted on the stereotaxic frame. Injections of 0.5 $\mu \mathrm{l}$ at a rate of $0.1 \mu \mathrm{l} / \mathrm{min}$ were made at four sites: anteroposterior (AP), +2.7; mediolateral (ML), \pm 0.7 ; dorsoventral (DV), -4.5 and AP, +3.7; $\mathrm{ML}, \pm 0.7 ; \mathrm{DV},-4.0$. After each injection, the needle was left in position for $10 \mathrm{~min}$ to allow absorption of the bolus and to minimize spread of the toxin along the needle tract.

Anterior cingulate cortex lesions were produced with injections of quinolinic acid (0.09 M; Sigma) made with a $2 \mu$ l Hamilton syringe mounted on the stereotaxic frame. Injections of 0.2 or $0.3 \mu \mathrm{l}$ at a rate of $0.1 \mu \mathrm{l} / \mathrm{min}$ were made at six sites within the anterior cingulate cortex: AP, $+2.2(0.2 \mu \mathrm{l})$; $\mathrm{ML}, \pm 0.5 ; \mathrm{DV},-2.4 ; \mathrm{AP},+2.7(0.3 \mu \mathrm{l}) ; \mathrm{ML}, \pm 0.5 ; \mathrm{DV}$, -2.6 and $\mathrm{AP},+3.2(0.2 \mu \mathrm{l})$; ML, \pm 0.5 ; DV,-2.4 . After each injection, the needle was left in position for $2 \mathrm{~min}$ to allow absorption of the bolus and to minimize spread of the toxin along the needle tract. This procedure and excitotoxin was chosen because this was found in pilot studies to be appropriate for minimizing extra-cingulate damage. Shamoperated controls $(n=12)$ received an identical procedure (one-half PFC coordinates, one-half ACC coordinates), with the exception that no neurotoxin was infused. After a minimum of 1 week of postoperative recovery, rats were gradually reduced to $85 \%$ of age-matched free weights.

\section{Histology}

After completion of testing, rats were given a lethal overdose of sodium pentobarbitone (Euthatal) and perfused with saline $(0.9 \%)$ and formal saline $(10 \%, w / v)$. Brains were removed and postfixed in formal saline and before cutting were transferred to a $25 \%$ sucrose solution where they remained for $24 \mathrm{~h}$. Slices ( $40 \mu \mathrm{m}$ thick) were made using a cryostat (Leica, Nussloch, Germany) and mounted onto gelatin-coated slides. These were subsequently dried, first at room temperature, then in an oven (temperature, $40^{\circ} \mathrm{C}$ ), before being stained with cresyl violet. The
Table 1. Experimental design for all animals

\begin{tabular}{lllll}
\hline \multirow{2}{*}{ Context } & Biconditional training & Congruent & Incongruent & Single element \\
\cline { 3 - 5 } $\mathrm{C} 1$ & $\mathrm{~A} 1: \mathrm{LP1} \rightarrow \mathrm{R} 1$ & $\mathrm{~A} 1 \mathrm{~V} 1, \mathrm{~A} 2 \mathrm{~V} 2$ & $\mathrm{~A} 1 \mathrm{~V} 2, \mathrm{~A} 2 \mathrm{~V} 1$ & $\mathrm{~A} 1, \mathrm{~A} 2$ \\
$\mathrm{C}$ & $\mathrm{A} 2: \mathrm{LP} 2 \rightarrow \mathrm{R} 1$ & & & \\
$\mathrm{~V} 1: \mathrm{LP} 1 \rightarrow \mathrm{R} 2$ & $\mathrm{~A} 1 \mathrm{~V} 1, \mathrm{~A} 2 \mathrm{~V} 2$ & $\mathrm{~A} 1 \mathrm{~V} 2, \mathrm{~A} 2 \mathrm{~V} 1$ & $\mathrm{~V} 1, \mathrm{~V} 2$ \\
& $\mathrm{~V} 2: \mathrm{LP} 2 \rightarrow \mathrm{R} 2$ & & & \\
\hline
\end{tabular}

$C 1 / C 2, R 1 / R 2, L P 1 / L P 2, A 1 / A 2$, and V1/V2 were different experimental chambers (contexts), reinforcers, lever presses, and auditory and visual stimuli, counterbalanced across animals.

extent and location of cell loss were verified with a light microscope and compared with the brain atlas of Paxinos and Watson (1998).

\section{Apparatus}

Eight operant chambers $(30 \mathrm{~cm}$ wide $\times 24 \mathrm{~cm}$ deep $\times 21 \mathrm{~cm}$ high; Med Associates, Georgia, VT), housed in sound-attenuating chambers and arranged in a two-by-four array, were used. They were positioned in a room that remained dark during the experiment. Each chamber consisted of three aluminum walls and ceiling, with a Perspex door serving as the fourth wall. The walls and ceiling were lined with transparent Perspex, behind which context cue "wallpapers" were fixed. Four of the chambers had wallpapers consisting of black and white checks, and the other four had white wallpaper with black spots. Each chamber had a floor constructed of 19 stainless-steel rods (3.8 $\mathrm{mm}$ in diameter, spaced $1.6 \mathrm{~cm}$ apart). The chambers were illuminated by a $3 \mathrm{~W}$ house light located at the top center of the left wall. Food pellets ( $45 \mathrm{mg}$; P. J. Noyes, Lancaster, $\mathrm{NH}$ ) could be delivered into a recessed magazine located in the right wall of each chamber. Twenty percent (w/v) sucrose solution (made up daily) could be delivered via a dipper into the same magazine. Access to the magazine was detected by means of infrared detectors mounted across the mouth of the recess. Two flat-panel retractable levers could be inserted to the left and right of the magazine. Two panel lights (diameter, $2 \mathrm{~cm}$ ) were also located in the right wall of the chamber, and a magazine light was sited in the top of the magazine and illuminated blue. Auditory stimuli consisted of a $2 \mathrm{kHz}$ tone and a $10 \mathrm{~Hz}$ train of clicks delivered from speakers located in the ceiling. Visual stimuli were flashing panel lights and steady panel lights plus the magazine light. A computer equipped with MED-PC software (Med Associates) controlled the operant chambers and recorded the data.

\section{Procedure}

Pretraining. On day one, all rats received two sessions of magazine training, one in each context, learning to retrieve both pellets and sucrose from the magazine. House lights were illuminated for the duration of each session and throughout all subsequent experimental sessions. Magazine training sessions lasted for $48 \mathrm{~min}$, and rats received reward approximately every $120 \mathrm{~s}$. After this, there were $2 \mathrm{~d}$ (four sessions, one in each context daily) of lever press training. During each of these sessions, the rats received 24 lever presentations (12 each of the right and the left lever, in random sequence), each lasting for $60 \mathrm{~s}$ and with an interlever interval of $60 \mathrm{~s}$. Session duration was again $48 \mathrm{~min}$. On the first day of lever press training, rats were rewarded on a continuous reinforcement schedule; this was altered on the second day to a random interval 15 (RI15) schedule, such that reward becomes available on average once in a $15 \mathrm{~s}$ period and the next lever press response will lead to delivery of a reward. This RI15 schedule remained in place for the remainder of the experiment. Animals then proceeded to biconditional discrimination training.

Biconditional discrimination training. Table 1 shows the experimental design for all animals. Rats were trained for a total of $14 \mathrm{~d}$, on two concurrent biconditional discriminations, with auditory and visual discriminative stimuli $\left(\mathrm{S}_{\mathrm{D}}\right)$, in the two contexts [checked and spotted $(\mathrm{C} 1$ and $\mathrm{C} 2)$ ]. Correct responses were rewarded with pellets in one context and sucrose in the other. In context C1 (checked), rats received presentations of the auditory cues [tone or clicks (A1 or A2)], during which the alternative lever presses [left or right (LP1 or LP2) ]led to reinforcement (R1; sucrose solution or pellets). Similarly, these lever presses (LP1 and LP2) led to the alternative reward (R2) in the presence of visual cues 
[steady or flashing lights (V1 and V2)] in context C2 (spotted). The contextual cues, auditory and visual stimuli, responses, and reinforcers were counterbalanced as far as was possible across animals. Rats received two sessions every day, one with each discrimination. Sessions consisted of 24 trials ( 12 of each trial type; A1 and A2 or V1 and V2) with a variable interstimulus interval (range, 45-75 s; mean, $60 \mathrm{~s}$ ). Discriminative stimulus presentations lasted $60 \mathrm{~s}$, reinforcement was unavailable during the first $10 \mathrm{~s}\left(\mathrm{~S}_{\mathrm{D}} 1\right)$, and was available during the final $50 \mathrm{~s}\left(\mathrm{~S}_{\mathrm{D}} 2\right)$ on a random interval $15 \mathrm{~s}$ reinforcement schedule. Both levers were present during each trial and were retracted during the interstimulus interval.

Extinction test sessions. After acquisition of the biconditional discriminations, rats received two test sessions, one in each training context. Test sessions consisted of presentations of the training stimuli (A1 and A2, V1 and V2) in addition to presentations of audiovisual compounds of the training stimuli (i.e., A1V1, A2V2, A1V2, and A2V1); these single stimuli trials were introduced to serve as comparisons for the test compounds. Stimulus presentations occurred in the presence of both levers but without the opportunity for reward. Rats received 24 extinction trials, four of each stimulus type, and $S_{D}$ duration was again $60 \mathrm{~s}$. Trial order was block randomized, with each trial type being presented once in each block of six trials. There was one test session per day, with the context in which rats were first tested counterbalanced across animals.

\section{Test stimuli}

The test stimulus compounds were classified as congruent or incongruent depending on the responses previously required during training (Table 1).

Congruent stimulus compounds. Congruent stimulus compounds were those that comprised elements that had been conditioned to elicit the same lever press response during acquisition. For the example given above, both stimulus elements of A1V1 and A2V2 compounds had elicited the same lever press responses in training (LP1 for A1 and V1; LP2 for $\mathrm{A} 2$ and $\mathrm{V} 2$ ).

Incongruent stimulus compounds. Incongruent stimulus compounds were those composed of elements that had been conditioned to elicit different lever press responses during initial training (e.g., A1V2, A2V1). Correct responses to each incongruent stimulus compound were designated based on the stimulus element that had been trained previously in the test context. Thus, if the test session occurred in the context in which the auditory discrimination had been acquired, then the auditory stimulus element governed the correct response; similarly, when the test was the context in which the visual discrimination had been learned, then the visual stimulus elements were the relevant cues. Using the explicit example from above, if the incongruent stimulus compound A1V2 was presented in context $\mathrm{C} 1$ (in which the auditory discrimination had been trained), then the correct response would be LP1, because this behavior had been associated previously with the stimulus element A1. If the same compound was presented in context $\mathrm{C} 2$, in which the visual discrimination had been acquired, then the correct response would be LP2, because this had been associated previously with the stimulus element V2. Thus, the test context was used to disambiguate the conflicting responses associated with the individual elements of the test compounds.

\section{Experiment 1b: the effect of reinforcer devaluation on contextual conditioning}

The aim of experiment $1 \mathrm{~b}$ was to examine whether the deficit in contextual control after prefrontal lesions observed in experiment la was a consequence of the failure of these animals to process or learn about contextual cues. This experiment investigated whether animals with lesions to the PFC and ACC had acquired the different context-outcome associations during training (i.e., C1-R1, C2-R2) using a specific-satiety reinforcer devaluation procedure. If $\mathrm{PFC}$ damage resulted in a generalized deficit in the ability to learn about or process contextual cues, then one might expect that these animals would be unable to demonstrate a selective reduction in responding to the contextual cues associated with the devalued reinforcer.
Subjects and apparatus

The subjects and apparatus were as described for experiment 1a.

\section{Procedure}

Retraining on biconditional discrimination tasks. After completion of experiment $1 \mathrm{a}$, rats were given $2 \mathrm{~d}$ of retraining on the original biconditional discrimination tasks; details were as described for experiment 1 a.

Extinction test sessions. After retraining on the biconditional discrimination tasks, rats received $2 \mathrm{~d}$ of extinction testing. Test days comprised two test sessions, one in each of the training contexts ( $\mathrm{C} 1$ and $\mathrm{C} 2)$. Test session duration was $15 \mathrm{~min}$, and the house light was illuminated for the duration of the session. Both levers were presented, in extinction, and responses (LP1 and LP2) were recorded. Before the test sessions, animals received a $1 \mathrm{~h}$ period of reinforcer devaluation by prefeeding. This was to examine the influence of reinforcer devaluation on instrumental responding to the training contexts.

Reinforcer devaluation. Devaluation consisted of a $1 \mathrm{~h}$ period of exposure to the reinforcer before test, during which the rats were able to feed to reach satiety. Devaluation exposures were conducted in the colony room, and rats were individually placed in cages with either $30 \mathrm{~g}$ of food pellets or $25 \mathrm{ml}$ of $15 \%$ sucrose solution in a drinking bottle. One-half of the animals were devalued with reinforcer R1, and one-half were devalued with R2. Consequently, for one-half of the animals, the reward associated with context $\mathrm{C} 1$ was devalued, and that associated with context $\mathrm{C} 2$ was nondevalued; the opposite was true for the remainder of the animals.

Immediately after each of the extinction tests, animals were allowed ad libitum access to both reinforcers simultaneously (R1 and R2, prefed and non-prefed), for $30 \mathrm{~min}$ in their home cage, and the overall consumption of each reinforcer was measured. The aim of this test was to evaluate the effectiveness of the devaluation procedure in the different groups.

\section{Results \\ Experiment 1a \\ Histology}

The aim of this experiment was to investigate the impact of lesions to the medial wall of the prefrontal cortex (incorporating both the prelimbic and anterior cingulate cortices); any animals that did not show $>50 \%$ damage to these regions were removed from this experiment. Consequently, four animals were removed from the PFC group because of limited cell loss within the prefrontal cortex. Figure 1 depicts the maximum (striped region) and minimum (gray region) extent and location of damage in the prefrontal cortex $(n=8)$. In all cases, rats exhibited substantial cell loss within the prelimbic and cingulate cortex subregions. Cell loss within the infralimbic cortex was more variable, but nevertheless present to some degree in all animals.

Because the ACC group were included in this experiment to enable comparison of the effects of ACC damage alone with those of a more general prefrontal lesion (ACC and prelimbic cortex damage), any animals in this group exhibiting damage extending to the prelimbic cortex were excluded. Hence, two rats exhibiting either minimal cell damage to the anterior cingulate cortex or damage to the prelimbic cortex were removed from the ACC lesion group. Figure 2 depicts the maximum (striped areas) and minimum (gray areas) extent of damage to the anterior cingulate cortex for the remaining animals $(n=10)$. Although animals exhibited minor cell loss within the secondary motor cortex, analysis of the behavioral data did not reveal a systematic relationship between extent of damage and behavioral measures, and so these animals were still retained. Two sham-operated controls that failed to acquire the biconditional discrimination tasks were also removed from the analysis $(n=10)$.

\section{Behavioral results}

Pretraining. All rats successfully learned to approach the magazine and to press levers to collect reward. 


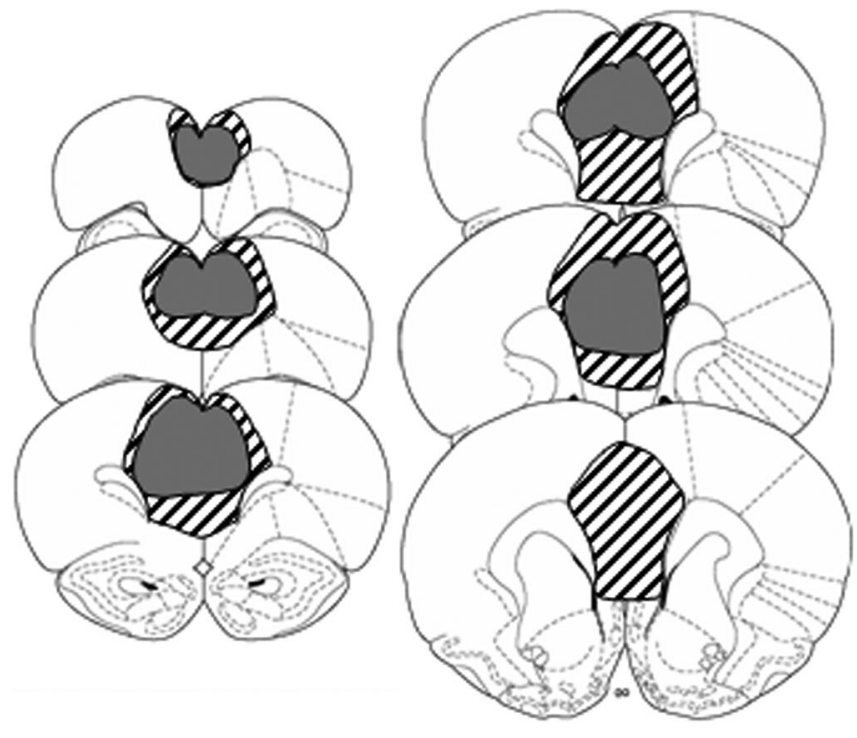

Figure 1. Representations of the minimum (gray) and maximum (striped) extent and location of damage within the prefrontal cortex. The outlines are reproduced from Paxinos and Watson (1998) and represent sections ranging from 2.2 to 4.7 anterior to bregma.
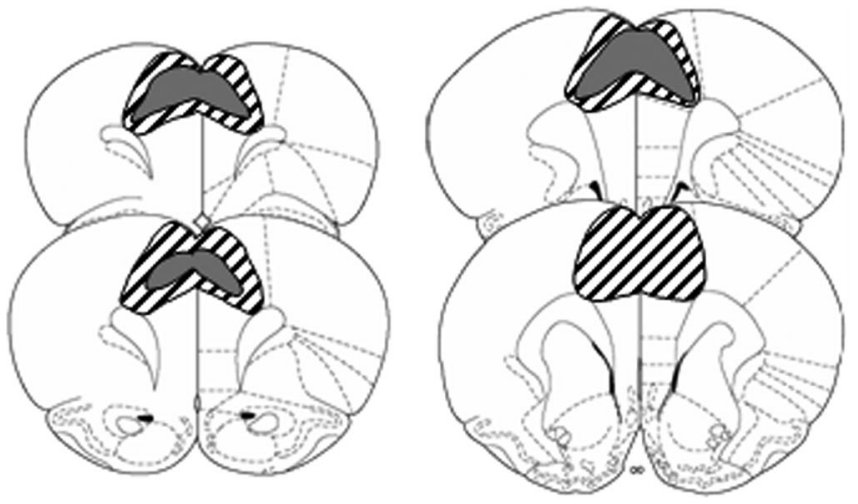

Figure 2. Representations of the minimum (gray) and maximum (striped) extent and location of damage within the anterior cingulate cortex. The outlines are reproduced from Paxinos and Watson (1998) and represent sections ranging from 2.2 to 3.7 anterior to bregma.

Acquisition of the biconditional discrimination tasks. Responses during the first $10 \mathrm{~s}$ of $\mathrm{S}_{\mathrm{D}}$ presentation were assessed and are shown in Figure 3. Reinforcement was unavailable during this period, so that the behavioral measure used to assess discrimination performance was uncontaminated by presentation of reward. Because similar patterns of responding were observed with performance on both the auditory and visual biconditional discrimination task, performance on the two tasks was averaged to produce an overall measure of biconditional discrimination task performance. All lesion groups (sham, ACC, and PFC) successfully acquired the biconditional discrimination tasks, with all animals producing more correct than incorrect responses by the end of training. A mixed ANOVA with a between-subjects factor of lesion (sham, ACC, PFC) and a within-subjects factor of session (numbers 1-14) was conducted on the acquisition data, revealing a main effect of session $\left(F_{(13,325)}=40.336 ; p<0.001\right)$ but no effect of group or any interaction of these factors (maximum $\left.F_{(2,25)}=1.686\right)$.

Test performance: responding to the biconditional training stimuli in extinction. Figure 4 shows the performance across the full $60 \mathrm{~s}$ stimulus duration for the three groups. Because observed

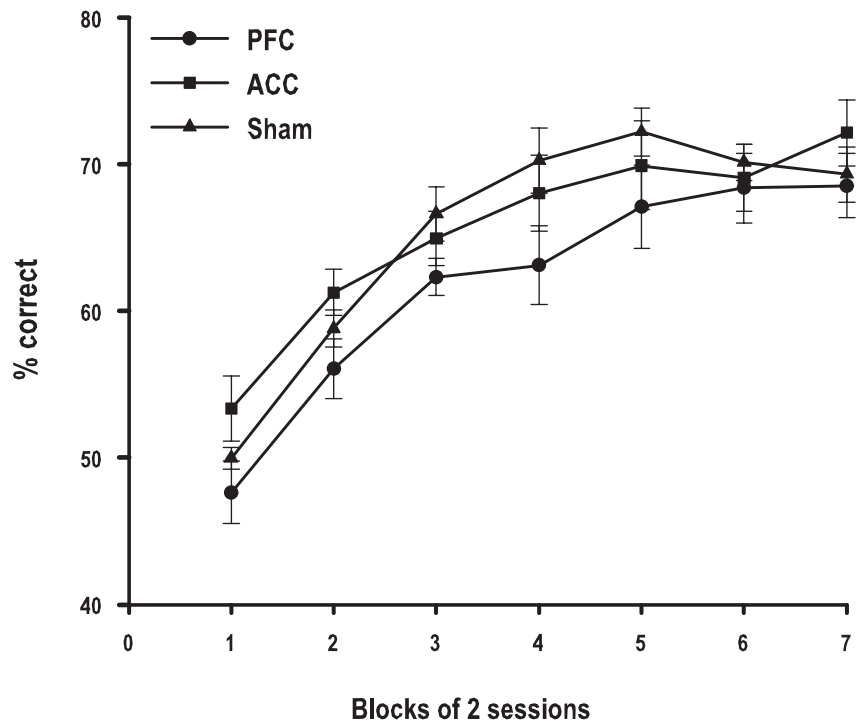

Figure 3. Normal acquisition of biconditional discrimination tasks in ACC- and PFC-lesioned animals. Error bars represent 1 SEM.

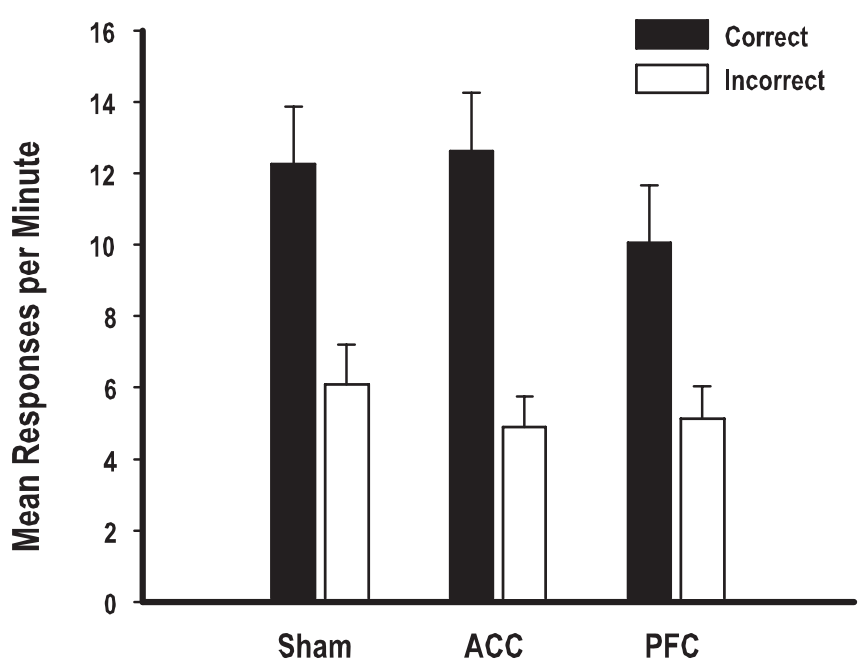

Figure 4. Accurate performance to the biconditional training stimuli in extinction in sham, ACC-lesioned, and PFC-lesioned animals. Error bars represent 1 SEM.

with biconditional discrimination performance during training, all three groups demonstrated good performance to the single element training stimuli in extinction, producing more correct than incorrect lever press responses. A mixed ANOVA with a between-subject factor of lesion (sham, ACC, PFC) and a withinsubjects factor of lever (correct, incorrect) confirmed this description of the data, revealing a main effect of lever $\left(F_{(1,25)}=\right.$ 94.913; $p<0.001$ ) but no effect of lesion nor any interaction of these factors (maximum $F_{(2,25)}=1.559$ ).

Test performance: responding to congruent and incongruent stimulus compounds. Because all stimuli and contexts were fully counterbalanced, the data from the test sessions were collapsed across stimuli and contexts, and average correct and incorrect response rates during congruent and incongruent stimulus compounds were calculated. Different patterns of responding were observed during $S_{D} 1$ (first $10 \mathrm{~s}$ of $S_{D}$ presentation) and $S_{D} 2$ (final $50 \mathrm{~s}$ of $\mathrm{S}_{\mathrm{D}}$ presentation) recording periods in animals with lesions of ACC and PFC, and data from these two recording periods are therefore presented and analyzed separately. 


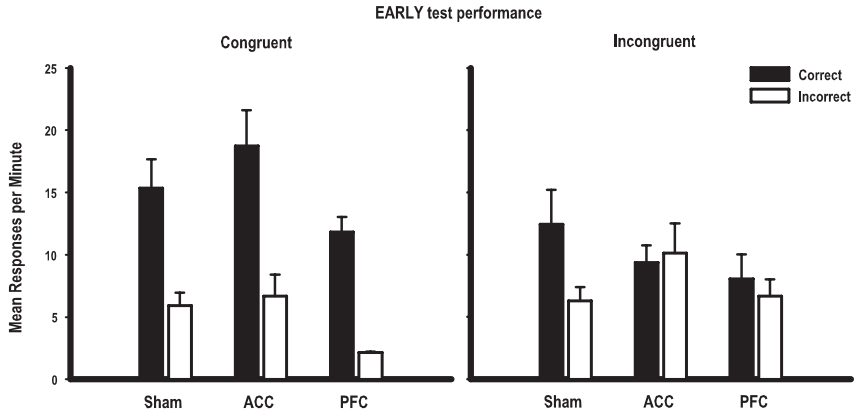

Figure 5. Abolition of context-appropriate responding to incongruent stimulus compounds in animals with PFC and ACC lesions, early (first $10 \mathrm{~s}$ ) in $\mathrm{S}_{\mathrm{D}}$ presentation. Congruent compounds comprised elements that required the same response during training; incongruent compounds comprised elements that required different responses during training. Error bars represent 1SEM.

Early test performance: $S_{D} 1$ responding. Figure 5 shows the average responses per minute to congruent and incongruent stimulus compounds during the first $10 \mathrm{~s}$ of $\mathrm{S}_{\mathrm{D}}$ presentation $\left(\mathrm{S}_{\mathrm{D}} 1\right.$ recording period).

All groups demonstrated good discrimination performance to the congruent stimulus compounds, responding more on the correct than the incorrect lever. Furthermore, sham-operated animals responded more on the correct than the incorrect lever during incongruent stimulus compounds. Hence, shamoperated rats biased their responding according to the stimulus element previously trained in the test context. However, this context-appropriate responding was not observed in animals with damage to either the ACC or PFC; during incongruent stimulus compounds, these rats failed to respond according to the stimulus element previously trained in the test context, pressing both levers at approximately equivalent rates.

A mixed ANOVA with a between-subjects factor of lesion (sham, ACC, PFC) and within-subjects factors of $\mathrm{S}_{\mathrm{D}}$ type (congruent, incongruent) and lever (correct, incorrect) revealed a main effect of lever $\left(F_{(1,25)}=61.020 ; p<0.001\right)$, an $\mathrm{S}_{\mathrm{D}}$ type-bylever interaction $\left(F_{(1,25)}=33.921 ; p<0.001\right)$, and a significant lesion-by- $S_{\mathrm{D}}$ type-by-lever interaction $\left(F_{(2,25)}=3.887 ; p<0.05\right)$. No other effects or interactions were significant (maximum $F_{(1,25)}=3.755 ; p=0.064$; main effect of $S_{D}$ type). Analysis of the simple effects of this three-way interaction revealed a lesion-bylever interaction for responding during incongruent stimulus compounds $\left(F_{(2,50)}=3.632 ; p<0.05\right)$ but not for responding during congruent stimulus compounds $(F<1)$. Correct and incorrect lever pressing differed during congruent stimulus compounds in all three lesion groups $\left(F_{(1,50)}>26.033 ; p<0.001\right)$, further demonstrating the successful acquisition of the biconditional discrimination tasks during training. Correct and incorrect lever pressing also differed during incongruent stimulus compounds in sham-operated animals $\left(F_{(1,50)}=11.026 ; p<0.05\right)$, demonstrating that these animals were able to perform the context-appropriate response when faced with ambiguous cues. Crucially, both ACC- and PFC-lesioned animals failed to show a significant difference in lever pressing during incongruent stimulus compounds $(F<1)$. Thus, although both ACC- and PFClesioned groups successfully acquired the biconditional discrimination tasks during training, neither was able to show contextappropriate responding during incongruent stimulus compounds during the first $10 \mathrm{~s}$ of $\mathrm{S}_{\mathrm{D}}$ presentation.

Late test performance: $S_{D} 2$ responding. Figure 6 shows the average correct and incorrect lever press rates during congruent and incongruent stimulus compounds over the final $50 \mathrm{~s}$ of $\mathrm{S}_{\mathrm{D}}$ pre-

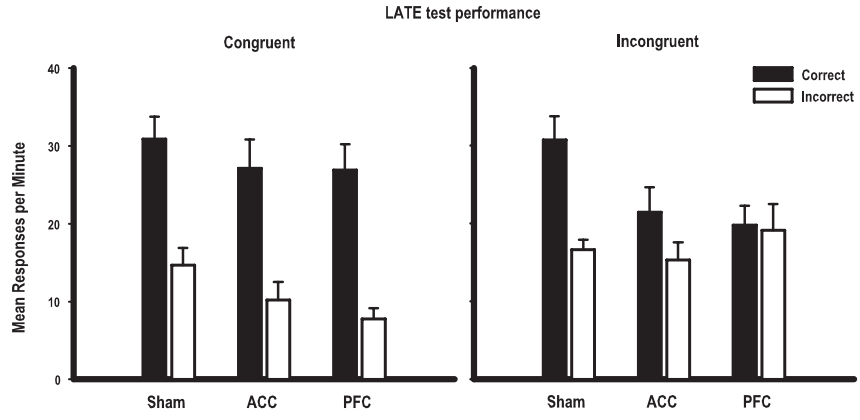

Figure 6. Abolition of context-appropriate responding to incongruent stimulus compounds only in animals with PFC lesions, late (final $50 \mathrm{~s}$ ) in $\mathrm{S}_{\mathrm{D}}$ presentation. Congruent compounds comprised elements that required the same response during training; incongruent compounds comprised elements that required different responses during training. Error bars represent 1 SEM.

sentation $\left(\mathrm{S}_{\mathrm{D}} 2\right.$ recording period) for the three lesion groups. As with responding early in $S_{D}$ presentation, accurate performance to congruent stimulus compounds is seen in all three lesion groups. Sham-lesioned animals also demonstrated good use of contextual cues to control responding during incongruent stimulus compounds, whereas PFC lesions appeared to abolish this influence of contextual cues. In contrast to performance early on in $\mathrm{S}_{\mathrm{D}}$ presentation, ACC-lesioned animals demonstrated greater context-appropriate than inappropriate responding during incongruent stimulus compounds, indicating that the use of contextual cues to control responding was at least partially intact in these animals.

A mixed ANOVA with a between-subjects factor of lesion (sham, ACC, PFC) and within-subjects factors of $\mathrm{S}_{\mathrm{D}}$ type (congruent, incongruent) and lever (correct, incorrect) revealed a main effect of lever $\left(F_{(1,25)}=98.077 ; p<0.001\right)$ and significant $\mathrm{S}_{\mathrm{D}}$ type-by-lever $\left(F_{(1,25)}=20.925 ; p<0.001\right)$ and lesion-by- $\mathrm{S}_{\mathrm{D}}$ type-by-lever interactions $\left(F_{(2,25)}=4.283 ; p<0.05\right)$. No other main effects or interactions were significant (maximum $F_{(2,25)}=$ 1.588). Simple effects analysis of the three-way interaction revealed a significant lesion-by-lever interaction for responding during incongruent stimulus compounds $\left(F_{(2,50)}=5.381 ; p<\right.$ $0.01)$ but not congruent stimulus compounds $(F<1)$. More specifically, correct and incorrect lever pressing differed during congruent stimulus compounds in all three lesion groups $\left(F_{(1,50)}<30.975 ; p<0.001\right)$ and also during incongruent stimulus compounds in sham-lesioned animals $\left(F_{(1,50)}=\right.$ 23.456; $p<0.001)$ and ACC-lesioned animals $\left(F_{(1,50)}=4.421\right.$; $p<0.05)$. However, no significant difference between correct and incorrect lever presses during incongruent stimulus compounds was observed in animals with PFC lesions $(F<1)$.

In summary, comparable correct responding by shamoperated and lesioned (both ACC and PFC) animals was observed during congruent stimulus compounds, indicating successful acquisition of the biconditional discrimination during training. Sham-operated animals also demonstrated correct performance on incongruent trials, revealing contextual control of biconditional discrimination performance. The absolute magnitude of this correct-incorrect difference was somewhat reduced compared with that observed during congruent trials. Animals with ACC lesions were impaired on incongruent trials during the initial period of incongruent compound presentations, but intact contextual control of responding emerged as each incongruent compound presentation progressed. In contrast, PFC-lesioned animals failed to demonstrate any contextual control of responding at any point during incongruent compound $S_{D}$ presentation, despite exhibiting normal performance during congruent trials. 


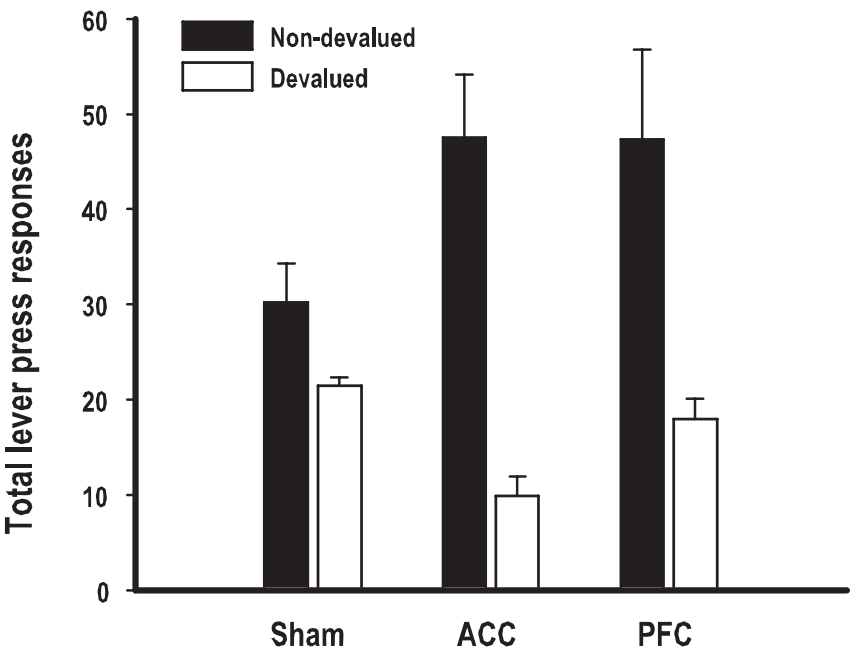

Figure 7. Normal effect of reinforcer devaluation on responding to contextual cues in ACCand PFC-lesioned animals. For the nondevalued condition, the devalued reinforcer had not been presented previously in the test context, whereas for the devalued condition, the devalued reinforcer had been presented previously in the test context. Error bars represent 1 SEM.

\section{Experiment 1b}

Retraining on the biconditional discrimination tasks

Animals in all three groups produced more correct than incorrect lever press responses on the last day of training (means \pm SEM: PFC lesion, $67 \pm 0.019 \%$ correct; ACC lesion, $70 \pm 0.022 \%$; sham, $67 \pm 0.015 \%)$. A between-subjects ANOVA with the factor of group conducted on this performance revealed no main effect of group $(F<1)$, indicating that all three groups were responding comparably to the biconditional discrimination stimuli.

Test performance: effect of reinforcer devaluation on instrumental responding to contextual cues

Because the variance of the data (total responses during the devaluation test) correlated with the means, a square-root transform was applied to the test data (Howell, 1997). Figure 7 shows the mean lever press responding after prefeeding with either a reinforcer previously presented in the test context (devalued condition) or a reinforcer that had not been presented previously in the test context (nondevalued condition). These lever press responses reflect the square of the transformed means from the analysis, in accordance with Howell (1997). Responding was found to be greater in the nondevalued condition compared with the devalued condition in sham, ACC-lesioned, and PFClesioned animals. A mixed ANOVA with a between-subject factor of group (ACC lesion, PFC lesion, sham) and devaluation condition (devalued, nondevalued) was conducted on the transformed means. A significant main effect of devaluation condition was revealed $\left(F_{(1,25)}=6.679 ; p<0.05\right)$, but no effects of group or any interaction were significant $(F<1)$. Hence, although both ACCand PFC-lesioned animals appeared to show a larger influence of reinforcer devaluation on lever press responding than did sham animals in Figure 7, this was not significant, and appears likely to be a consequence merely of increased variability observed in the ACC and PFC lesion groups. Thus, all animals demonstrated a similar pattern of responding to contextual cues after reinforcer devaluation, indicative of all three groups having acquired associations between the context and reinforcer type during training.

Test performance: consumption tests

Responding during consumption tests was averaged across sessions and reinforcers because a similar pattern was observed in all conditions. Consumption tests revealed decreased consumption of the prefed reinforcer in all animals (means \pm SEM: PFC lesion: prefed, $10.638 \pm 0.39 \mathrm{~g}$; non-prefed, $1.486 \pm 0.34 \mathrm{~g}$; ACC lesion: prefed, $11.008 \pm 0.64 \mathrm{~g}$; non-prefed, $2.408 \pm 0.33 \mathrm{~g}$; sham: prefed, $9.940 \pm 0.78 \mathrm{~g}$; non-prefed, $1.097 \pm 0.18 \mathrm{~g})$. A mixed ANOVA with a between-subject factor of group (ACC lesion, PFC lesion, sham) and prefeeding (prefed, non-prefed) revealed a significant effect of prefeeding $\left(F_{(1,25)}=488.015 ; p<0.001\right)$ but no effect of group $\left(F_{(2,25)}=2.562 ; p=0.098\right)$ nor any interaction $(F<1)$. The results from the consumption tests indicate that any effects seen in the context devaluation sessions could not have been a consequence of a differential ability to demonstrate reinforcer-specific satiety in lesioned or sham-lesioned animals.

\section{Discussion}

The experiments reported here provide evidence that the rat prefrontal cortex, like that of the human, is central to the performance of goal-directed choice behavior, in particular in situations of response conflict. Using a novel task that mimics some aspects of the cue and response conflict frequently used in cognitive control tasks in humans experiment 1a demonstrated that sham-operated animals were able to show context-appropriate responding to incongruent stimulus compounds. Rats used the contextual cues experienced during training to disambiguate the response conflict induced by presentation of incongruent stimulus compounds. However, this context-appropriate responding was abolished in PFC-lesioned animals and was transiently impaired in ACC-lesioned animals during the initial $10 \mathrm{~s}$ period of incongruent stimulus presentation; context-appropriate responding emerged during the final $50 \mathrm{~s}$ of incongruent cue presentations in animals with ACC lesions.

The abolition of context-appropriate responding in rats with lesions to the PFC was not likely to be a consequence of a failure to learn stimulus-response associations during initial biconditional discrimination training, because responding during congruent and single-element trials was comparable with that seen in sham-operated animals (experiment 1a). Nor was this disruption on incongruent trials caused by a failure to learn about, or process, contextual cues per se. ACC-lesioned animals were able to make use of contextual cues during the final $50 \mathrm{~s}$ of incongruent stimulus presentations (experiment 1a) (Fig. 4), and both ACCand PFC-lesioned animals demonstrated appropriate response devaluation attributable to context-outcome associations in experiment $1 \mathrm{~b}$. Consequently, the selective impairment in responding during incongruent stimulus compounds in both PFClesioned and ACC-lesioned animals appears restricted specifically to the explicit use of contextual cues as task-setting stimuli that come into play when animals are presented with incongruent cue compounds that lead to competing responses.

The finding that the contextual control of responding to incongruent stimulus compounds is impaired after PFC damage is consistent with research implicating the human prefrontal cortex in task-appropriate responding in the Stroop task and similar assays of cognitive control. For example, patients with frontal damage have difficulty performing the Stroop task and display increased reaction times and error rates when selecting the subordinate but task-appropriate response (i.e., color naming) over the dominant tendency of word reading on incongruent trials. (Perret, 1974; Cohen and Serven-Schrieber, 1992). In fact, the results of experiment 1a suggest a specific role for the prefrontal cortex in enhancing task-appropriate responding, rather than inhibiting task-inappropriate responses; PFC lesions were found to reduce correct responses during incongruent stimulus com- 
pounds rather than to increase incorrect responses. This finding agrees with the view of conflict control behavior proposed by Cohen and colleagues (Cohen et al., 1990; Cohen and ServenSchrieber, 1992; Miller and Cohen, 2001), which suggests that the PFC is vital for enhancing activation within task-appropriate stimulus-response pathways, rather than inhibiting activation within task-inappropriate pathways.

Following alternative characterizations of aspects of frontal function, the results are also consistent with a role for this region in behavioral flexibility in response to novel or confusing situations, in the active maintenance of a task or goal (working memory) and in situations in which novel distracters have to be ignored (Cohen et al., 1990; Ragozzino et al., 1999a,b, 2003; Delatour and Gisquet-Verrier, 2000; Rougier et al., 2005). For example, it has been suggested that the frontal-dependent enhancement of task-relevant pathways may not only necessitate the maintenance of task-relevant information over time (Cohen et al., 1998), but also permit appropriate responses to be made in the face of competing, perhaps more compelling (i.e., as a result of novelty), alternative behavioral choices.

The fact that prefrontal lesions impaired contextual control of responding during incongruent compounds suggests that this effect is unlike the contextual modulation of simple associations that is disrupted by damage to the hippocampal formation [e.g., impaired context-specific latent inhibition (Honey and Good, 1993) and impaired background contextual conditioning (Phillips and LeDoux, 1994; Rudy and Sutherland, 1995; O'Reilly and Rudy, 2001)]. Rather, this PFC-dependent control involves rulebased performance in which contextual, or task-setting, cues are used as the basis of higher-order rules to enable the appropriate control of choice responses. Indeed, we have direct evidence that, unlike PFC lesions, excitotoxic damage to the hippocampal formation in the rat does not disrupt the contextual control of performance in this task (Haddon and Killcross, unpublished data). Consequently, the novel procedure used in experiment 1a provides an animal model of frontally mediated cognitive control of response conflict by task-setting cues and so permits the direct and detailed examination of the neurochemical and neuroanatomical systems underpinning this function. It is also of interest to note that experiment $1 \mathrm{~b}$ provided evidence that contextual cues were able to mediate outcome devaluation, suggesting that transfer effects can depend on the current value of instrumental outcomes, in contrast to some previous research (Rescorla, 1994).

Some researchers (Duncan and Owen, 2000) have argued that there is little strong evidence for functional specialization within the prefrontal cortex, suggesting that smaller lesions to this region would result in smaller impairments compared with deficits seen with larger amounts of damage. Although it is possible that the results from animals with ACC lesions might be explained by this type of mass action hypothesis, one might suppose that a smaller impairment would be observed in ACC-lesioned animals but that this deficit would be evident across the entire duration of the stimulus presentation. This was not the case. The deficit observed during the early phase of stimulus presentation was as profound in animals with ACC damage as that seen in animals with greater PFC damage but dissociated significantly during the later stages of incongruent trials. Moreover, a more detailed examination of the relationship between lesion size and location and performance during incongruent stimulus compounds (Haddon and Killcross, 2005) has confirmed that a mass action hypothesis is unlikely to be able to account for the findings after differential PFC damage. Consequently, although there is some damage to the cingulate cortex common to both the ACC- and PFC-lesioned groups, and this damage may contribute in part to the deficit in contextual control seen with larger PFC lesions, ACC damage does not appear sufficient to produce the complete disruption of responding seen in PFC-lesioned animals. Thus, it seems plausible that the more complete abolition of behavioral control by task-setting contextual cues seen with large PFC lesions may also be a consequence of the extensive damage to the prelimbic region of the medial prefrontal cortex observed in these animals. Such a specific role for the prelimbic cortex in goaldirected choice behavior is consistent with previous findings (Corbit and Balleine, 2003; Coutureau and Killcross, 2003; Killcross and Coutureau, 2003), and we have preliminary data suggesting that reversible inactivation of the prelimbic PFC by the $\mathrm{GABA}_{\mathrm{A}}$ agonist muscimol produces a profound deficit in the use of task-setting contextual cues (Killcross et al., 2005).

In human neuroimaging studies, the ACC has often been found to be active during performance of incongruent trials. The interpretation of these findings has, however, been clouded by observations of the activation of this region during performance of congruent trials (MacDonald et al., 2000; Milham et al., 2003a,b). The results of experiment 1a provide some additional evidence for a necessary role for the ACC in processes that are engaged during incongruent trials, in particular those processes that operate at the start of incongruent stimulus presentation. This finding is in accordance with theories implicating the ACC in response-related processes such as the detection of response conflict (Carter et al., 1998, 2000; Botvinick et al., 1999) or in "selection for action" processes (Frith et al., 1991). These theories suggest that the ACC promotes the exertion of greater cognitive control in situations in which increased control is required, such as at the point of decision or choice (Rushworth et al., 2004). Because the ACC impairment was observed only during the initial $10 \mathrm{~s}$ period of stimulus presentation, the low rates of responding during this first $10 \mathrm{~s}$ (one to two responses) make it likely that the observed impairment is perhaps limited to preliminary choice responses rather than some preliminary time period. Although they do not directly address these specific potential functions of the ACC, the results presented here agree with this general property of the ACC, demonstrating decreased correct responding early on in incongruent stimulus presentation as a result of a failure to enhance activation within task-relevant pathways rapidly after the detection of error or conflict.

In summary, pretraining excitotoxic lesions to the PFC and the ACC resulted in deficits in a task designed to assess contextual control of cue and response conflict in rats. In particular, ACC lesions temporarily impaired this contextual control of instrumental responding, and larger PFC lesions abolished it completely. This study also provides additional evidence for the role of the PFC in behavioral choice, implicating this area in the processes by which cues and rules come to control behavior in the face of stimulus and response competition.

\section{References}

Botvinick M, Nystrom LE, Fissell K, Carter CS, Cohen JD (1999) Conflict monitoring versus selection-for-action in anterior cingulate cortex. Nature 402:179-181.

Carter CS, Braver TS, Barch DM, Botvinick MM, Noll D, Cohen JD (1998) Anterior cingulate cortex, error detection, and the online monitoring of performance. Science 280:747-749.

Carter CS, Macdonald AM, Botvinick M, Ross LL, Stenger VA (2000) Parsing executive processes: strategic vs. evaluative functions of the anterior cingulate cortex. Proc Natl Acad Sci USA 97:1944-1948.

Cohen JD, Serven-Schreiber D (1992) Context, cortex, and dopamine: a 
connectionist approach to behavior and biology in schizophrenia. Psychol Rev 99:45-77.

Cohen JD, Dunbar K, McClelland JL (1990) On the control of automatic processes: a parallel distributed processing account if the Stroop effect. Psychol Rev 97:332-361.

Cohen JD, Braver TS, O'Reilly RC (1998) A computational approach to prefrontal cortex, cognitive control and schizophrenia: recent developments and current challenges. In: The prefrontal cortex: executive and cognitive functions (Roberts AC, Robbins TW, Weiskrantz L, eds), pp 195-220. Oxford: Oxford UP.

Corbit LH, Balleine BW (2003) The role of the prelimbic cortex in instrumental conditioning. Behav Brain Res 146:145-157.

Coutureau E, Killcross S (2003) Inactivation of the infralimbic cortex reinstates goal-directed responding in overtrained rats. Behav Brain Res 146:167-174.

Delatour B, Gisquet-Verrier P (2000) Functional role of rat prelimbicinfralimbic cortices in spatial memory: evidence for their involvement in attention and behavioral flexibility. Behav Brain Res 109:113-128.

Duncan J, Owen AM (2000) Common regions of the human frontal lobe recruited by diverse cognitive demands. Trends Neurosci 23:475-483.

Frith CD, Friston KJ, Liddle PF, Frackowiak RSJ (1991) Willed action and the prefrontal cortex in man: a study with PET. Proc R Soc Lond B Biol Sci 244:241-246.

Haddon JE, Killcross AS (2005) Medial prefrontal cortex lesions abolish contextual control of competing responses. J Exp Anal Behav 84:485-504.

Honey R, Good M (1993) Selective hippocampal lesions abolish the contextual specificity of latent inhibition and conditioning. Behav Neurosci 107:23-33.

Howell DC (1997) Statistical methods for psychology, Ed 4 (Hinrichs C, ed), pp 327-328. Belmont, CA: Duxbury.

Killcross AS, Coutureau E (2003) Coordination of actions and habits in the medial prefrontal cortex of rats. Cereb Cortex 13:400-408.

Killcross AS, Marquis J-P, Haddon JE (2005) GABA $_{\mathrm{A}}$ agonist injection in prelimbic cortex, but not in infralimbic cortex, impairs contextual control of response conflict in rats. Behav Pharmacol 16:S27.

MacDonald AW, Cohen JD, Stenger VA, Carter CS (2000) Dissociating the role of dorsolateral prefrontal cortex and anterior cingulate cortex in cognitive control. Science 288:1835-1838.

MacLeod CM (1991) Half a century of research on the Stroop effect: an integrative review. Psychol Bull 109:163-203.

Milham MP, Banich MT, Claus ED, Cohen NJ (2003a) Practice-related effects demonstrate complementary roles of the anterior cingulate and prefrontal cortices in attentional control. NeuroImage 18:483-493.
Milham MP, Banich MT, Barad V (2003b) Competition for priority in processing increases prefrontal cortex's involvement in top-down control: an event-related FMRI study of the Stroop task. Brain Res Cogn Brain Res 17:212-222.

Miller EK, Cohen JD (2001) An integrative theory of prefrontal cortex function. Annu Rev Neurosci 24:167-202.

O’Reilly RC, Rudy JW (2001) Conjunctive representations in learning and memory: principles of cortical and hippocampal function. Psychol Rev 108:311-345.

Paxinos G, Watson C (1998) The rat brain in stereotaxic coordinates, Ed 4. San Diego: Academic.

Perret E (1974) The left frontal lobe of man and the suppression of habitual responses in verbal categorical behaviour. Neuropsychologia 12:323-330.

Phillips R, LeDoux J (1994) Lesions of the dorsal hippocampal formation interfere with background but not foreground contextual fear conditioning. Learn Mem 1:34-44.

Ragozzino ME, Wilcox C, Raso M, Kesner RP (1999a) Involvement of rodent prefrontal cortex subregions in strategy switching. Behav Neurosci 113:32-41.

Ragozzino ME, Detrick S, Kesner RP (1999b) Involvement of the prelimbic cortex in behavioural flexibility for place and response learning. J Neurosci 19:4585-4594.

Ragozzino ME, Kim J, Hassert D, Minniti N, Kiang C (2003) The contribution of the rat prelimbic-infralimbic areas to different forms of taskswitching. Behav Neurosci 117:1054-1065.

Rescorla RA (1994) Transfer of instrumental control mediated by a devalued outcome. Anim Learn Behav 22:27-33.

Rosvold HE, Mirsky AF, Saranson I, Bransome ED, Beck LH (1956) A continuous performance test of brain damage. J Consult Psychol 20:343-350.

Rougier NP, Noelle D, Braver TS, Cohen JD, O’Reilly RC (2005) Prefrontal cortex and the flexibility of cognitive control: rules without symbols. Proc Natl Acad Sci USA 102:7338-7343.

Rudy J, Sutherland R (1995) Configural association theory and the hippocampal formation: an appraisal and reconfiguration. Hippocampus 5:375-389.

Rushworth MF, Walton ME, Kennerley SW, Bannerman DM (2004) Action sets and decisions in the medial prefrontal cortex. Trends Cogn Sci 8:410-417.

Stroop JR (1935) Studies of interference in serial verbal reactions. J Exp Psychol 18:643-662.

Wylie G, Allport A (2000) Task switching and the measurement of "switch costs." Psychol Res 63:212-233. 